\title{
Evaluation der DFG-Nachwuchsakademie „Klinische Studien“ - Publikationen und Folgeförderungen
}

\author{
Levka Dahmen ${ }^{1}$, Frank Krummenauer ${ }^{1}$
}

\section{Zusammenfassung}

Hintergrund I Die Nachwuchsakademie „Klinische Studien“ ist ein Förderinstrument der Deutschen Forschungsgemeinschaft (DFG) zur Reaktion auf den im Bereich der klinischen Forschung zunehmenden Mangel an qualifizierten Nachwuchskräften. Die Bewerbung um Teilnahme an der Nachwuchsakademie basiert auf einem selbst vorgeschlagenen Akademie-Projekt, für dessen Durchführung bei der DFG eine Anschubförderung beantragt werden kann. Nach vier Ausrichtungsrunden der Nachwuchsakademie sollte untersucht werden, welche Publikations- und Drittmittel-Leistungen seitens der Teilnehmer aus diesen Akademie-Projekten generiert werden konnten.

Methoden | Die 92 Teilnehmer aller vier bisher von der DFG 2008-2012 unterstützten Ausrichtungsrunden wurden anhand eines standardisierten Erhebungsbogens adressiert. Erfasst wurden die aus den Akademie-Projekten erwachsenen Publikationsprofile, die damit generierte ImpactFaktor-Summe sowie die erwirkte kumulative Folgefördersumme.

Ergebnisse I Insgesamt nahmen 64 der 92 Teilnehmer an der Befragung teil. Von 41 umgesetzten Projekten waren 32 DFG-seitig gefördert worden, woraus von 13 Autoren 27 Publikationen mit einer kumulativen Impact-Faktor-Punktzahl von 130 veröffentlicht wurden. 21 der 64 Teilnehmer stellten einen Antrag auf Folgeförderung ihres Akademie-Projektes; davon erhielten 13 Antragsteller eine Förderung im kumulierten Umfang von $5223000 €$.

Schlussfolgerung | Obgleich nur jeweils 13 von 64 Befragten die Generierung von Impact-Faktorbeladenen Veröffentlichungen respektive Folgeförderungen gelang, zeigten sich diese auf für wissenschaftlichen Nachwuchs sehr ermutigendem Niveau.

\section{Einleitung}

In der human- und zahnmedizinischen klinischen Forschung ist seit Jahren ein wachsender Mangel an qualifizierten Nachwuchswissenschaftlern zu verzeichnen [1]. Die speziellen Anforderungen an den akademischen Nachwuchs in der klinischen Forschung ergeben sich aus ihrer interdisziplinären Stellung zwischen klinischer und grundlagenorientierter Arbeit. Insbesondere für die patientenorientierte Forschung werden Ärzte benötigt, die über ein umfassendes Verständnis in der grundlagen- und krankheitsorientierten Forschung verfügen. Daraus abgeleitete Erkenntnisse im Umgang mit Patienten, Krankheiten und Therapieansätzen können bestätigt und deren Wirksamkeit im klinischen Praxisalltag überprüft und kritisch abgewogen werden $[1,2]$. Die enge Zusammenarbeit dieser Bereiche und die interdisziplinäre Qualifizierung von in Praxis und Forschung geschulten Wissenschaftlern sichern langfristig die Qualität und Sicherheit einer leistungsfähigen Krankenversorgung und machen nachhaltige medizinische Spitzenforschung in Deutschland möglich [3,4].
Für eine Vielzahl junger Ärzte hat der berufliche Weg in die klinische Forschung in den vergangenen Jahren jedoch an Attraktivität verloren. Grund hierfür sind die starke Verdichtung der klinischen Arbeit, die deutlich schlechtere Bezahlung wissenschaftlicher Tätigkeit, die nur bedingt mögliche Anerkennung von Forschungszeiten für die Weiterbildungszeit und nicht zuletzt die Finanzierungs-bedingt oft befristeten Stellenangebote innerhalb der klinischen Forschungslandschaft in Deutschland [5-7]. Unter diesen Konditionen sind viele junge Mediziner trotz eines aktiv bekundeten Interesses an wissenschaftlicher Arbeit nicht bereit, einen Einstieg in die klinische Forschung zu wagen [8].

Als eine konstruktive Reaktion auf diese Situation wurde seitens der Deutschen Forschungsgemeinschaft (DFG) erstmalig im Jahr 2008 die Ausrichtung der DFG-Nachwuchsakademie „Klinische Studien“ unter Federführung eines in der Praxis klinischer Studien erfahrenen Forschers am Standort Gießen/Nauheim gefördert. Es folgten drei weitere Ausrichtungsrunden in den Jahren 2009, 2010 und 2012 an den Medizinischen Fakultäten der Universitäten Mannheim, Würzburg und Witten/Herdecke. Die Bewerbungsmöglich-
Institute

1 Institut für Medizinische Biometrie und Epidemiologie, Universität Witten/ Herdecke, Witten

\section{Korrespondenz}

B.A. cand. med.

Levka Dahmen

Institut für Medizinische

Biometrie und Epidemiologie

Fakultät für Gesundheit der

Universität Witten/Herdecke

Alfred Herrhausen-Straße 50

58448 Witten

Tel: 02302926766

Fax: 0230292644785

Levka.Dahmen@uni-wh.de 
keit um Förderung einer Ausrichtungsrunde der DFG-Nachwuchsakademie „Klinische Studien“ war schon für die erste Ausrichtungsrunde 2008 offen für alle Medizinischen Fakultäten in Deutschland. Die jeweiligen Ausrichter haben dann zu den entsprechenden Ausrichtungsrunden fakultätsseitig individuelle Anträge auf Förderung ihrer lokalen Ausprägungen der Nachwuchsakademien gestellt. Die dritte Ausrichtungsrunde begann im Oktober 2010, die vierte erst im Januar 2012 im Interesse einer hinreichend langen Bewerbungsfrist zur Teilnahme.

Ansatz dieses DFG-Förderinstruments ist die strategische Förderung und Qualifizierung junger ärztlicher und zahnärztlicher Nachwuchskräfte bezüglich methodischer, regulatorischer und praktischer Belange klinischer Studien. Basis ist eine eigene Projektidee („Akademie-Projekt“), die bei der Bewerbung um die Teilnahme eingereicht wird. Die Umsetzung dieses einjährigen, durch die DFG als Sachbeihilfe förderbaren Projektes soll die Vorbereitung einer umfangreichen Nachfolge-Förderantragstellung ermöglichen: Beispielsweise kann es Inhalt des „Akademie-Projektes“ sein, eine umfangreiche multizentrische Studie bezüglich Logistik (Zentrenrekrutierung etc.), Methodik (Endpunkt- und Messinstrumenten-Auswahl, statistische Planung) und Regulatorik (Antragstellung an Ethik-Kommissionen und Überwachungsbehörden) vorzubereiten. Die Folgeförder-Antragstellung kann dann auf die Finanzierung dieser klinischen Studie selbst abzielen.

Die DFG stellt die Mittel zur Durchführung der Akademie bereit, inklusive Reise- und Unterbringungskosten für die ausgewählten Teilnehmer. Im Anschluss an die Teilnahme werden nach erfolgreicher Antragstellung einzelne Akademie-Projekte von der DFG mit bis zu $60000 €$ gefördert. In Absprache mit dem Förderer wurde beschlossen, das Instrument über alle vier Ausrichtungsrunden 2008-2012 zu evaluieren. Betrachtet wurden die aus den Akademie-Projekten entstandenen Publikationen und Folgeförderungen. Die Ergebnisse sollen Aufschluss darüber geben, welche mittelfristigen Effekte die Nachwuchsakademie „Klinische Studien“ erzielt und inwiefern hierdurch der Einsatz von finanziellen Ressourcen mit Ergebnissen gerechtfertigt wurden.

\section{Methodik}

Insgesamt wurden im Zeitraum von 2008-2012 vier DFG-Nachwuchsakademien „Klinische Studien“ ausgerichtet. Fester Bestandteil der Bewerbung von interessierten Nachwuchswissenschaftlern war die Skizze eines eigenen Forschungsvorhabens. Die einwöchige Akademie selbst war als Intensivschulung mit Seminaren zu methodischen, regulatorischen und praktischen Aspekten klinischer Studien konzipiert. Zudem bestand die Mög- lichkeit, das individuelle Forschungsvorhaben als „Akademie-Projekt“ vorzustellen und mithilfe eines Mentoring weiter zu entwickeln. Im Anschluss konnten die Teilnehmer einen Antrag auf Förderung dieses „Akademie-Projektes“ an die DFG stellen. Die vier Akademie-Ausrichtungen wurden programmatisch vergleichbar ausgerichtet. Einzig in der vierten Ausrichtungsrunde wurde im Anschluss an die Intensivschulung mit einer DFG-Referentin vor Ort eine fakultative Beratung zur Antragstellung an die DFG angeboten.

Die Datenerhebung erfolgte in Form einer anonymisierten Befragung aller Teilnehmer aus den Ausrichtungsrunden 1-4. Hierfür wurde ein Erhebungsbogen zunächst postalisch versandt; bei ausbleibender Antwort wurde bis zu zweimal elektronisch nachgesandt. Rückfragen von Teilnehmern oder Implausibilitäten in deren Angaben wurden elektronisch oder telefonisch geklärt. Der Fragebogen enthielt zum einen Fragen zur Förderung, Finanzierung und Strukturierung der von den Teilnehmern umgesetzten „Akademie-Projekte“. Zum anderen wurden die daraus generierten Publikationen und Folgefördermittel erfragt.

Von den insgesamt 92 Teilnehmern entfielen auf die Ausrichtungsrunden 1-4 jeweils 22, 25, 25 und 20 Personen. Mit Stichtag zum 8.9.2015 wurde ein anonymisierter Datensatz aus den 64 erhaltenen Rückläufen (70\%) generiert, umfassend 13, 19, 14 respektive 18 Teilnehmer der Ausrichtungsrunden $1-4$.

Die Angaben der Teilnehmer zu den aus „Akademie-Projekten“ resultierenden Veröffentlichungen wurden via PubMed überprüft. Alle Publikationen konnten aufgefunden werden, stellten zudem Ergebnisse durchgeführter Projektideen dar und konnten dem Bereich der klinischen und patientenbezogenen Forschung zugeordnet werden. Weiter wurde der für die Fachzeitschrift im jeweiligen Bezugsjahr geltende Impact Faktor festgehalten und daraus im Anschluss pro Teilnehmer die Summe der aus dem „Akademie-Projekt“ generierten Impact-Faktor-Punktzahl (IP) gebildet.

Primäre Endpunkte der Evaluation waren die pro „Akademie-Projekt“ berichtete Publikationsanzahl, die damit generierten kumulierten IP sowie die (aus dem Projekt abgeleitete) kumulative Folgefördersumme.

Die Ausrichtungsrunden wurden mit Blick auf die explorative Natur der Evaluation nur deskriptiv gegenüber gestellt: Die Erfassung und statistische Auswertung der quantitativen Daten wurde mit IBM SPSS ${ }^{\circledR}$ Statistics 22 für Windows ${ }^{\circledR}$ durchgeführt. Als Lagemaße wurden der Median- sowie die totale Summe der Publikationsprofile und Fördervolumina bestimmt. Die Auswertung von Freitextangaben erfolgte manuell in die 


\begin{tabular}{|c|c|c|c|c|c|}
\hline Parameter & $\begin{array}{l}\text { 1. Akademie } \\
(2008)\end{array}$ & $\begin{array}{l}\text { 2. Akademie } \\
(2009)\end{array}$ & $\begin{array}{l}\text { 3. Akademie } \\
(2010)\end{array}$ & $\begin{array}{l}\text { 4. Akademie } \\
(2012)\end{array}$ & Gesamt \\
\hline Teilnehmer der Evaluation & 13 & 19 & 14 & 18 & 64 \\
\hline durchgeführte* Akademie-Projekte & 9 & 11 & 12 & 9 & 41 \\
\hline \multicolumn{6}{|c|}{ Anträge auf Anschubfinanzierung an die DFG (Akademie-Projekte) } \\
\hline gestellte Anträge & 11 & 14 & 12 & 8 & 45 \\
\hline bewilligte Anträge & 8 & 8 & 9 & 7 & 32 \\
\hline \multicolumn{6}{|l|}{ Publikationen aus Akademie-Projekten } \\
\hline publizierende Personen & 4 & 5 & 2 & 2 & 13 \\
\hline Anzahl Publikationen & 9 & 12 & 3 & 3 & 27 \\
\hline \multicolumn{6}{|c|}{ Folgeförderungen aus Akademie-Projekten } \\
\hline Anträge auf Folgeförderung & 7 & 6 & 5 & 3 & 21 \\
\hline bewilligte Folgeförderungen & 5 & 4 & 2 & 2 & 13 \\
\hline
\end{tabular}

Feedback-Kategorien „positiv“ / „negativ“ / „konstruktiv“. Abschließend wurden per manueller Item-Analyse übergeordnete Themenkomplexe herausgearbeitet.

\section{Ergebnisse}

Von insgesamt 92 Teilnehmern antworteten bis zum Stichtag der Evaluation 64 Befragte (70\%). 45 der 64 Evaluierten gaben an, einen Antrag auf Anschubförderung bei der DFG gestellt zu haben. 32 dieser 45 berichteten eine DFG-Förderung des geplanten „Akademie-Projektes“ erhalten zu haben; weitere 9 Teilnehmer berichteten zudem, mit andersseitiger Förderung ihr eigenes Projekt durchgeführt oder zum Zeitpunkt der Befragung begonnen zu haben $(\triangleright$ Tab. 1$)$.

Aus den 41 durchgeführten Projekten (irrespektive der Förderquelle) waren von 13 AkademieTeilnehmern insgesamt 27 Publikationen in wissenschaftlichen Zeitschriften mit Impact Faktor entstanden, darunter 18 unter Erstautorschaft der Teilnehmer. Für die vier Ausrichtungsrunden ergab sich jeweils eine kumulierte IP von 64,43, 44,45, 10,93 und 10,14; im Median lag die aus den 41 umgesetzten Akademie-Projekten generierte Impact-Faktor-Summe in allen Runden bei
0IP ( Tab. 2). Nur die 32 DFG-geförderten Teilnehmer wiesen Publikationen nach. Aus den 9 DFG-unabhängig finanzierten Projekten entstanden keine Publikationen.

Von den 64 Befragten berichteten ferner insgesamt 21 (33\%), auf Basis des geplanten Akademie-Projektes einen Folgeförderantrag gestellt zu haben (davon 54, 32, 36 und 17\% in den Ausrichtungsrunden 1-4); hierbei wurde viermal das gemeinsame Förderprogramm „Klinische Studien“ der DFG und des Bundesministeriums für Bildung und Forschung (BMBF) adressiert. Insgesamt 13 dieser 21 Folgeantragsteller (jeweils 5, 4, 2 und 2 Teilnehmer der vier Ausrichtungsrunden) berichteten den Erhalt einer Folgeförderung. Bewilligt wurden für die 21 Folgeantragsteller im Median 108000, 165000, 0 und $190000 €$. Kumuliert wurden Folgeförderungen in Höhe von $5223000 €$ eingeworben ( Tab. 3). Hierbei konnten 4 der 32 DFG-seitig geförderten Teilnehmer sowohl IP als auch Folgeförderungen aus dem geförderten Akademie-Projekt generierten.

Die qualitative Auswertung der Freitextkommentare ergab bei insgesamt 32 verwertbaren Beiträgen 18 positive, 11 negative und 3 konstruktive Kommentare. 12 der 18 positiven Kommentare bezogen sich explizit auf die „zielgerichtete Förde-
Tab. 1 Ergebnisse der ersten vier Ausrichtungsrunden der DFG-Nachwuchsakademie „Klinische Studien“.

\begin{tabular}{|lllll}
\hline Jahr & $\begin{array}{l}\text { publizierende } \\
\text { Personen }\end{array}$ & $\begin{array}{l}\text { Anzahl } \\
\text { Publikationen }\end{array}$ & summierte IP & mediane IP \\
\hline 1. Akademie (2008) & 4 & 9 & 64,43 & 0 \\
\hline 2. Akademie (2009) & 5 & 12 & 44,45 & 0 \\
\hline 3. Akademie (2010) & 2 & 3 & 10,93 & 0 \\
\hline 4. Akademie (2012) & 2 & 3 & 10,14 & 0 \\
\hline Summe & $\mathbf{1 3}$ & $\mathbf{2 7}$ & $\mathbf{1 2 9 , 9 5}$ & $\mathbf{0}$
\end{tabular}

Tab. 2 Aus den AkademieProjekten generierte kumulierte und mediane Impact-Faktor-Punktzahl (IP) der 41 EvaluationsTeilnehmer, die unabhängig von der DFG-seitigen Förderentscheidung ein geplantes Akademie-Projekt umgesetzt haben. 


\begin{tabular}{|lll|}
\hline Jahr & $\begin{array}{l}\text { kumulierte Folge- } \\
\text { fördersummen }(€)\end{array}$ & $\begin{array}{l}\text { im Median bewilligte } \\
\text { Folgefördersummen (€) }\end{array}$ \\
\hline 1. Akademie (2008) & 2506000 & 108000 \\
\hline 2. Akademie (2009) & 1610000 & 165000 \\
\hline 3. Akademie (2010) & 217000 & 0 \\
\hline 4. Akademie (2012) & 890000 & 190000 \\
\hline Summe & $\mathbf{5 2 2 3 0 0 0}$ & $\mathbf{1 0 0 0 0 0}$ \\
\hline
\end{tabular}

Tab. 3 Aus den AkademieTeilnahmen generierte Folgeförderungen der 21 Evaluations- Teilnehmer, die einen Antrag auf Folgeförderung gestellt haben. rung der eigenen Karriere-Richtung klinische Forschung durch die Teilnahme an der Nachwuchsakademie“. Die 11 negativen und die 3 konstruktiven Beiträge bezogen sich hauptsächlich auf kommunikative Missverständnisse in der Strukturierung der Akademie. Sie kommentierten u.a., dass mit dem verwendeten Erhebungsbogen sekundär durch die Teilnahme an der Akademie entstandenen Nutzen nicht abgebildet wurden. Dazu zählte z.B. das Sammeln wichtiger Erfahrungen für weitere Forschungsprojekte.

\section{Diskussion}

Nach zum Teil 7 Jahre zurückliegender Förderzusage konnten für nur 13 der 41 (30\%) umgesetzten Akademie-Projekte Impact-beladene Veröffentlichungen identifiziert werden. Auffällig ist, dass alle 27 Veröffentlichungen den DFG-geförderten Akademie-Projekten zugeschrieben werden konnten. Die Betrachtung der publikatorischen Leistung zeigt bezogen auf die 41 durchgeführten Projekte mit in allen Ausrichtungsrunden im Median 0 publizierten IP ein ernüchterndes Ergebnis. Die 13 DFG-geförderten Teilnehmer aber, die tatsächlich Publikationen erwirkten, taten dies sehr erfolgreich und mit einer für Nachwuchswissenschaftler beachtlich hohen IP-Summe. Gründe für die fehlende Publikationsleistung der restlichen durchgeführten Akademie-Projekte könnten beispielsweise an der generischen Natur einiger Projekte liegen (z.B. reine „logistische Vorbereitung“ einer in der Folgeförderung angestrebten klinischen Studie). Zudem muss berücksichtigt werden, dass 8 der 32 geförderten Akademie-Projekte sich zum Zeitpunkt der Befragung noch in der Umsetzungsphase befanden und zwangsläufig noch nicht publiziert werden konnten.

13 der 64 Teilnehmer gaben unabhängig von der Förderstruktur eine im Anschluss an die Umsetzung ihres Akademie-Projektes erfolgreich angestrebte Folgeförderung des eigenen Forschungsvorhabens an. Dies geschah in 4 Fällen aufgrund einer bereits fortgeschrittenen Projektplanung ohne eine DFG-Anschubfinanzierung direkt im Anschluss an die Akademieteilnahme. Ursachen dafür, dass von 64 Teilnehmern nur 21 Anträge (13 bewilligt) auf Folgeförderung stellten, könnten in der Struktur und Systematik der Akademie bestehen: Bereits während der Akademie-Veranstaltung wurde einigen Teilnehmern aus methodischen oder praktischen Gründen von der Umsetzung ihres Akademie-Projektes abgeraten. Im Falle der 41 umgesetzten Projekte muss ferner in Betracht gezogen werden, dass bei „negativen“ Ergebnissen des Akademie-Projektes ein Antrag auf Folgefördermittel von den Verantwortlichen als nicht mehr sinnvoll erachtet wurde. Abschließend sollte auch hier die Anzahl der noch in der Umsetzungsphase befindlichen Projekte Berücksichtigung finden.

Im Vergleich der vier Ausrichtungsrunden fällt ein zeitlicher Gradient sowohl in der kumulativ generierten publikatorischen Leistung als auch in der erzielten Folgefördersumme auf. Je früher die Ausrichtungsrunde zurück liegt, desto höher ist der erzielte Output. Dies verdeutlicht einmal mehr, dass zur Messung eines adäquaten Wirkungsgrades von Maßnahmen zur akademischen Nachwuchsförderung die Verwertungszeit nicht zu unterschätzen ist - und damit der Zeitpunkt der vorliegenden Befragung möglicherweise zumindest für die vierte Ausrichtungsrunde des Jahres 2012 noch zu früh terminiert gewesen sein mag.

Zur Überprüfung der Repräsentativität der evaluierten Kohorte bezüglich der zugrundeliegenden Gesamtheit aller Teilnehmer der vier Nachwuchsakademien wurden die Verteilung des Geschlechtes sowie die Förderquote verglichen: Die evaluierten Teilnehmer waren zu $41 \%$ weiblich gegenüber einem Anteil von $43 \%$ aller 92 Teilnehmer. Ferner wurden in beiden Kollektiven 50\% der Akademie-Projekte von der DFG gefördert. Limitierend für die Aussagekraft der Ergebnisse ist außerdem die Tatsache, dass die Angaben der Teilnehmer zum Vorliegen und zur Höhe eingeworbener Folgefördervolumina kaum validierbar waren. Zum verwendeten Erhebungsinstrument selbst geht aus den Freitextkommentare hervor, dass durch die Fokussierung der Evaluation auf einen quantifizierbaren Nutzen nur ein Teileffekt der Nachwuchsakademie abgebildet wurde: Alleine die Teilnahme an einer solchen Akademie kann für das Fachwissen und den persönlichen Lebenslauf einen - in einigen Fällen sogar belegbaren - essenziellen Schritt in der Karriereführung zum „Clinician Scientist“ bedeuten.

\section{Konsequenz für Klinik und Praxis}

- Nur ein moderater Anteil der Teilnehmer der bisherigen Ausrichtungsrunden der DFG-Nachwuchsakademie „Klinische Studien“ konnte in dieser Erhebung Publikationen oder Folgeförderungen aus dem in der Akademie im Vordergrund stehenden Akademie-Projekt vorweisen.

- Wenn jedoch eine akademische Verwertung erwirkt werden konnte, zeigte sich diese auf - für den von wissenschaftlichem Nachwuchs zu erwartendem Maßstab sehr ermutigendem Niveau. 


\section{Danksagung}

Die Autoren danken herzlich Herrn Dr. Frank Wissing (ehemals DFG, jetzt Medizinischer Fakultätentag) und Frau Christiane Krämer (DFG) für zahlreiche hilfreiche Rücksprachen aus Sicht des Förderers während der Konzeption und Umsetzung dieser Untersuchung. Ferner danken die Autoren Frau Dr. Ursula Hahn (Institut für Medizinische Biometrie und Epidemiologie der Universität Witten/Herdecke) für zahlreiche konstruktive Hinweise zur Berichterstellung der Ergebnisse im Rahmen der Dissertationsschrift von Levka Dahmen sowie Frau Dragana Mitric (Institut für Medizinische Biometrie und Epidemiologie der Universität Witten/Herdecke) für wertvolle logistische Unterstützung in der Durchführung der Erhebung.

\section{Literatur}

1 Meybohm P, Lindau S, Schürholz T et al. Wissenschaftlicher Beitrag: Förderung des wissenschaftlichen Nachwuchses durch medizinische Fachgesellschaften - Umfrage unter den

Mitgliedsgesellschaften der Arbeitsgemeinschaft der Wissenschaftlichen Medizinischen Fachgesellschaften e.V. (AWMF). Z Evid Fortbild Qual Gesundhwes 2015; 109: 632 - 641

2 Deutsche Forschungsgemeinschaft. Klinische Forschung - Denkschrift. Weinheim: Wiley-VCH; 1999

3 Siewert JR, Niethammer D. Klinische Forschung in Deutschland: Zu den Problemen der patientenorientierten Forschung und der Durchführung von klinischen Studien in den Deutschen Universitätsklinika. Chirurg 2003; 74: 1-3

4 Bundesministerium für Bildung und Forschung, Deutsche Forschungsgemeinschaft, Wissenschaftsrat. Hochschulmedizin der Zukunft: Ziele und Visionen für die klinische Spitzenforschung
(2004). http://www.dfg.de/download/pdf/ dfg_im_profil/reden_stellungnahmen/ 2005/hochschulmedizin_der_zukunft_ kernforderungen_bmbf_dfg_wr_klinische forschung_05.pdf (letzter Zugriff: 16.8.2016)

5 DGIM. Mitteilungen der DGIM. Internist 2012; 53: 1218-1229

6 Deutsche Forschungsgemeinschaft. Empfehlungen der Ständigen Senatskommission für Grundsatzfragen in der Klinischen Forschung der Deutschen Forschungsgemeinschaft (DFG) zur Weiterentwicklung der klinischen Forschung an der deutschen Universitätsmedizin in den Jahren 2015 - 2025. http://www.dfg.de/download/pdf/dfg_im_profil/ reden_stellungnahmen/2015/sgkf_empfehlungen_ klinische_forschung_150720.pdf (letzter Zugriff: 16.8.2016)

7 Deutsche Forschungsgemeinschaft. Etablierung eines integrierten Forschungs- und WeiterbildungsProgramms für „Clinical Scientists“ parallel zur Facharztweiterbildung. http://www.dfg.de/ download/pdf/dfg_im_profil/reden stellungnahmen/2015/empfehlungen_clinician_ scientists_0415.pdf (letzter Zugriff: 18.6.2016)

8 Else Kröner-Fresenius-Stiftung. Rahmenbedingungen medizinischer Forschung in Deutschland. http://www.ekfs.de/fileadmin/customer/images/ Publikationen/pdf/2014_Rahmenbedingungen_ medizinischer_Forschung_in_DE.pdf (letzter Zugriff: 16.8.2016)

\section{Anmerkung}

Teile der hier präsentierten Ergebnisse werden als Poster mit publiziertem abstract im Rahmen der Jahrestagung 2016 der Deutschen Ophthalmochirurgie (DOC) sowie im Rahmen der Jahrestagung 2016 der Deutschen Gesellschaft für Medizinische Informatik, Biometrie und Epidemiologie (GMDS) angemeldet. Ferner werden die in dieser Arbeit präsentierten Ergebnisse Teil der Dissertationsschrift von Levka Dahmen (cand. med. an der Universität Witten / Herdecke) zur Erlangung des Grades "Dr. med. « der Fakultät für Gesundheit Universität Witten/Herdecke sein.
Interessenkonflikt

Die Evaluation wurde seitens der DFG durch eine 6-monatige Finanzierung einer wissenschaftlichen Mitarbeiterstelle (50\% Vollzeitäquivalent) für Frau Dahmen unterstützt.

DOI 10.1055/s-0042-115777 Dtsch Med Wochenschr 2016; 141: e213-e217 (c) Georg Thieme Verlag KG . Stuttgart · New York . ISSN 0012-0472 